\title{
Adaptive Beamforming for OFDM Based Hybrid Mobile Satellite System
}

\author{
Ammar H. Khan, Muhammad A. Imran, Barry G. Evans \\ Centre for Communication Systems Research (CCSR) \\ University of Surrey, Guildford, Surrey GU2 7XH, United Kingdom \\ Email: $\{$ A.H.Khan, M.Imran, B.Evans\}@ surrey.ac.uk
}

Keywords: Hybrid mobile satellite, OFDM, Beamforming, Channel Estimation.

\begin{abstract}
Global connectivity cannot be guaranteed by terrestrial networks due to the lack of infrastructure in rural areas. Neither can satellite networks assure this due to lack of signal penetration and capacity coverage issues in densely populated areas. To bridge this gap, we propose an Orthogonal Frequency Domain (OFDM) based hybrid architecture where users are provided service by existing mobile networks in urban areas and are served by satellite in the rural areas. In such a system terrestrial and satellite networks can reuse the portion of spectrum dedicated to each of these systems resulting in significant increase in overall capacity, wider coverage and reduced cost. This frequency reuse induces severe Co-Channel Interference (CCI) at the satellite end and our work focuses on its mitigation using OFDM based adaptive beamforming.
\end{abstract}

\section{Introduction}

The evolution of multimedia services and applications has driven research for higher capacity communication networks with advanced antenna systems signal processing as a favourable solution. However, higher data rates alone do not produce an efficient communication network and neither can it guarantee a rich customer base. The task of providing service to people "whenever needed" and "wherever needed" is an attribute of a future successful communication network.

The need for such global connectivity puts significant pressure on the networks. Stand-alone terrestrial systems cannot guarantee this due to lack of infrastructure in rural areas. Neither can satellite networks guarantee this due to lack of signal penetration and capacity coverage issues in densely populated areas. This motivates the consideration of location/demand-based hybrid networks, where terminals can enjoy terrestrial coverage in urban areas and be served by satellite links as they move to rural areas. In such a system terrestrial and satellite networks can also reuse the portion of spectrum dedicated to each system resulting in significant increase in overall capacity. The service provisioning by two different technologies should be transparent to the end-user, in the sense that the same mobile terminals should work with both terrestrial as well as satellite networks. Enabling of such transparency would require a powerful satellite receiving end in order to ensure required link margin. Such a complimentary and transparent architecture offers lower cost, higher data rates, increased network capacity and wider coverage. This hybrid architecture with frequency reuse introduces severe CCI, which is a major impediment to the realisation of high capacity systems. Owing to the nature of hybrid systems, CCI from the mobile to the satellite becomes more significant and thus dominates the system performance.

A Code Division Multiple Access (CDMA) based hybrid architecture with similar structure has been pursued by Mobile Satellite Ventures (MSV) [4]. The MSV architecture provides coverage in rural areas via the satellite network and in urban areas via Ancillary Terrestrial Components (ATCs) but does not utilise the existing terrestrial networks. To enable transparency two satellites are deployed and space diversity is used to ensure the required link margin [5]. To mitigate CCI introduced by transparency, CDMA based adaptive beamforming $[5,12]$ is proposed. However, demand of higher data rates has motivated research for 4th Generation (4G) terrestrial networks. These networks, such as the one proposed by 3GPP LTE are based on OFDM due to its higher bandwidth efficiency and robustness against Inter Symbol Interference (ISI). For a hybrid network to offer $4 \mathrm{G}$ services in a transparent and seamless manner, the technology used for the satellite and terrestrial coverage must be similar, if not identical. Moreover, using adaptive beamforming in an OFDM based system yields better performance due to the possibility of using different beamforming weights for each sub-carrier. Hence for more robustness, higher capacity and compatibility with next generation high data rate networks, an OFDM based mobile satellite architecture will be needed in the future.

For an OFDM system, broadly two categories of adaptive beamforming are found in the literature applied in terrestrial system, Pre-Fast Fourier Transform (Pre-FFT) [6] and Post-Fast Fourier Transform (Post-FFT) beamforming [9]. However most of the work in the literature is focused on terrestrial systems. There is some work for satellite scenarios [4, 5, 12] but it is focused on CDMA based system. Most of the work relating to terrestrial scenarios assumes AWGN channel conditions. Others considering practical 


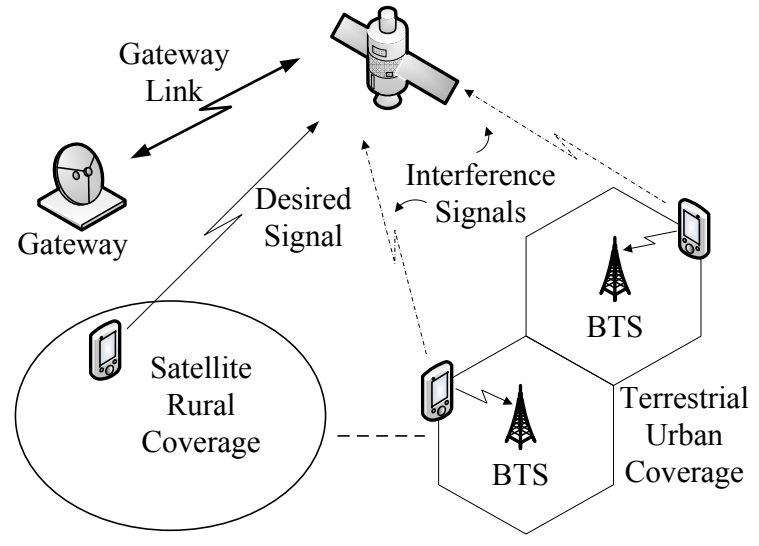

Figure 1: Hybrid System Scenario

channel models neither relate to the mobile satellite scenario $[1,7,10,8]$ nor to moderate or high user mobility. In the case of frequency selective channel models, there is an interplay between beamforming and channel estimation. Their inter-relation is an area yet to be explored. This paper focuses on the investigation of proposed OFDM based hybrid mobile satellite architecture in satellite channel scenarios and to study the interaction between beamforming and channel estimation specific to the satellite scenario.

\section{Hybrid Mobile Satellite System Model}

In this section, we discuss the proposed hybrid architecture and the system model devised to investigate the system performance.

\subsection{Hybrid Mobile Satellite Scenario}

The system scenario depicted in Figure 1 is of the OFDM based hybrid architecture discussed in the previous section. It can be seen that users in rural area are served directly by satellite spot beams while those in urban areas utilise the existing terrestrial networks. In this way, satellite networks need no additional infrastructure of their own to support users in cities. On the other hand terrestrial networks need not consider the service provisioning outside cities; this is taken care of by the satellite. Both networks utilise each others spectrum in their respective region of operation resulting in higher capacity, true global coverage and considerable reduction in cost.

With respect to this system, the main task is to mitigate uplink CCI that is induced by mobile terminals due to reuse of satellite link frequencies and we focus on the uplink from the perspective of the satellite. Each link from terminal to satellite is modelled as Single Input Multiple Output (SIMO). There are a total of $J$ users in the system, with one desired user being served by the satellite and the rest interfering users being served by the terrestrial network. The transmitted signal passes through a multi-tap time selective mobile satellite wireless channel. At the satellite end, PreFFT based adaptive beamforming is used to mitigate uplink interference. We assume that no pilot or frequency offsets exist in the system and the users utilise the same frequency band for transmission hence they completely overlap in the frequency domain.

\subsection{OFDM System Model}

Figure 2 illustrates the block diagram of the OFDM system with beamforming at the satellite end. At first random data is generated which is followed by QPSK modulation. The data is mapped together with pilots at known locations. Taking one symbol at a time, the output in frequency domain of a multi-user scenario can be represented as:

$$
\tilde{\mathbf{x}}_{j}=[\tilde{x}(1, j), \tilde{x}(2, j), \ldots, \tilde{x}(N, j)]^{T}
$$

where $\tilde{x}(n, j)$ represents the $n^{t h}$ sub-carrier of the $j^{t h}$ user, $n=1,2, \ldots, N, j=1,2, \ldots, J$ and $(\cdot)^{T}$ denotes the transpose operation. $\tilde{\mathbf{x}}_{j}$ denotes $j^{\text {th }}$ column of matrix $\tilde{\mathbf{X}}$. The OFDM symbol is then transformed into the time domain which can be expressed as:

$$
\mathbf{x}_{j}=\mathbf{F}^{H} \tilde{\mathbf{x}}_{j}
$$

where $\mathbf{x}_{j}=[x(1, j), x(2, j), \ldots, x(N, j)]^{T}$ is the OFDM symbol in time domain and,

$$
\mathbf{F}=\left[\begin{array}{cccc}
1 & 1 & \cdots & 1 \\
1 & e^{-j 2 \pi(1)(1) / N} & \cdots & e^{-j 2 \pi(1)(N-1) / N} \\
\vdots & \vdots & \ddots & \vdots \\
1 & e^{-j 2 \pi(N-1)(1) / N} & \cdots & e^{-j 2 \pi(N-1)(N-1) / N}
\end{array}\right]
$$

F represents the FFT operation matrix and $(\cdot)^{H}$ represents the Hermitian transpose. After transformation of the signal to the time domain, a cyclic prefix is appended where last $G$ elements of the OFDM symbol are copied to the start. This is done to combat ISI. Using Equation (2), the guard interval insertion process can be represented as:

$$
\overline{\mathbf{x}}_{j}=\left[\begin{array}{c}
\mathbf{I}_{N, G} \\
\mathbf{I}_{N}
\end{array}\right] \mathbf{F}^{H} \tilde{\mathbf{x}}_{j}
$$

where $\overline{\mathbf{x}}_{j}=[x(N-G+1, j), x(N-G+2, j), \ldots, x(N-$ $1, j), x(N, j), x(1, j), x(2, j) \ldots, x(N, j)]^{T}$ is the final OFDM symbol to be transmitted. $\mathbf{I}_{N, G}$ contains the last $G$ rows of size $N$ identity matrix $\mathbf{I}_{N}$. The output $\overline{\mathbf{x}}_{j}$ is passed to the Parallel-to-Serial $(\mathrm{P} / \mathrm{S})$ convertor. Here data is transformed to serial format, $\overline{\mathbf{x}}_{j}[k]$, and is then transmitted over the multi-tap time selective wireless channel. The effect of the channel can be presented as:

$$
\overline{\mathbf{y}}_{j}[k]=\overline{\mathbf{x}}_{j}[k] \otimes \mathbf{h}_{j}[k]
$$

Here $k$ denotes the time index. After passing through the channel, signals from desired and interference sources are received. The received signal matrix for one OFDM symbol at the satellite antenna elements, after removal of cyclic prefix, can be expressed as:

$$
\mathbf{V}=\mathbf{A} \mathbf{Y}^{H}+\mathbf{B}
$$



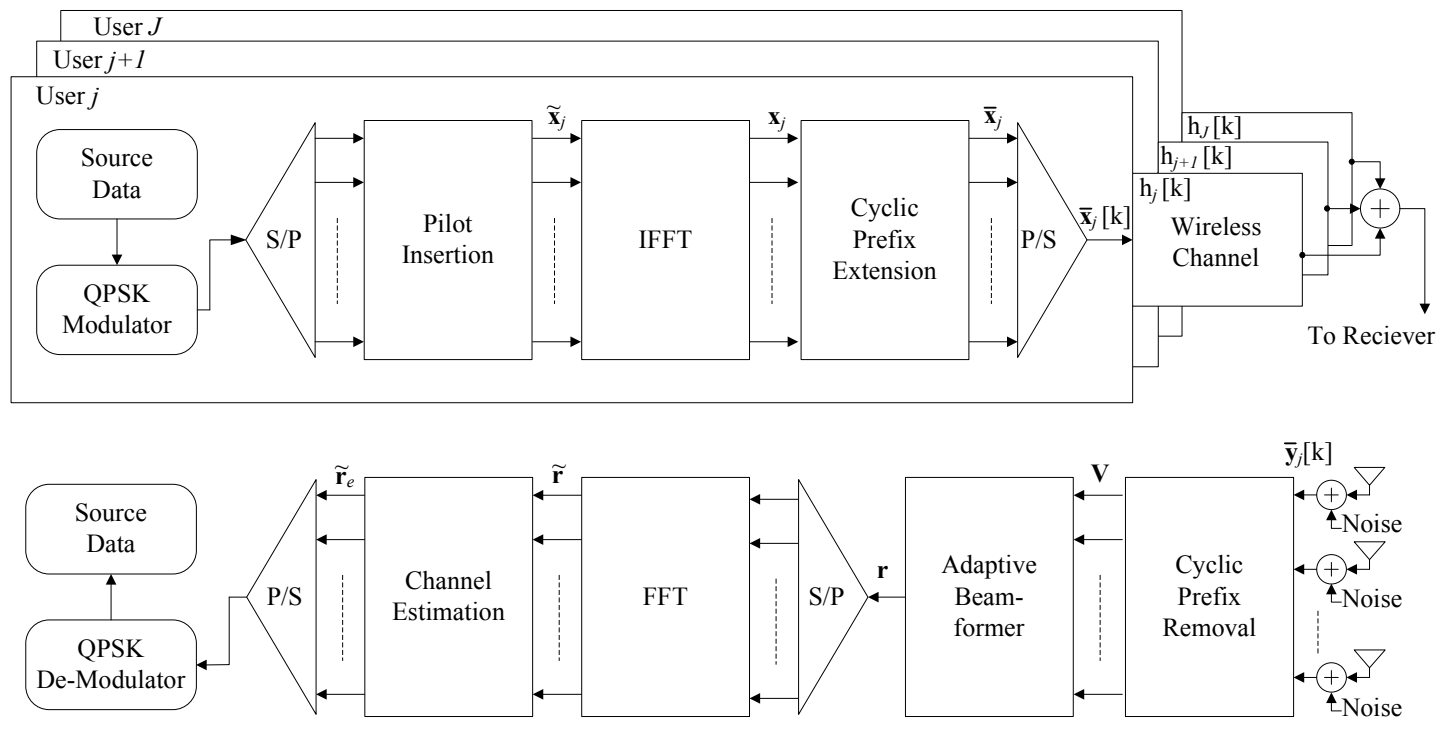

Figure 2: OFDM System Model

Here, $a(s, j)$ is the array response for the $s^{t h}$ antenna elements and $j^{\text {th }}$ user of matrix $\mathbf{A}$ is given as $e^{-j 2 \pi(s-1) d_{a} \sin \left(\theta_{j}\right) / \lambda} . s=1,2, \ldots, S$ are the total number of satellite antenna elements, $d_{a}$ is the inter-element spacing, $\theta_{j}$ is the Director-of-Arrival (DOA) of the $j^{t h}$ user and $\lambda$ is the carrier wavelength. We model a linear array hence the inter-element spacing $d_{a}$ is $\lambda / 2 . y(s, j)$ is the received signal for the $s^{\text {th }}$ antenna element and $j^{\text {th }}$ user of matrix $\mathbf{Y}$. Similarly $b(s, n)$ and $v(s, n)$ is the noise, and beamformer's output at $s^{\text {th }}$ elements and $n^{\text {th }}$ OFDM sub-carrier of matrix $\mathbf{B}$ and $\mathbf{V}$ respectively. Noise is independent and identically distributed Gaussian with zero mean and $\sigma^{2}$ variance.

\subsection{Adaptive Beamforming with Channel Estimation}

To mitigate CCI, the output of the array is processed by the adaptive beamformer where complex weights are applied, which can be written as:

$$
\mathbf{r}=\mathbf{w}^{H} \mathbf{V}
$$

where $\mathbf{r}=[r(1), r(2), \ldots, r(N)]$ is the weighted ouput of the beamformer and $\mathbf{w}=[w(1), w(2), \ldots, w(S)]^{T}$ are the complex weights for the antenna elements. This is followed by Serial-to-Parallel (S/P) conversion and transformation of beamformer output to the frequency domain, which can be expressed as:

$$
\tilde{\mathbf{r}}=\mathbf{F r}^{H}
$$

Here $\tilde{\mathbf{r}}=[\tilde{r}(1), \tilde{r}(2), \ldots, \tilde{r}(N)]^{T}$ is the received OFDM symbol in the frequency domain. The proposed architecture utilises a single weight per antenna element in the multipath fading environment, in which different fading exists across OFDM sub-carriers. Hence $\tilde{\mathbf{r}}$ cannot be decoded directly and channel estimation is essential to alleviate performance degradation. Several channel estimation techniques can be employed [2]. In our purposed architecture, we employ the Least Squares (LS) algorithm. Representing the channel transfer function at the pilot locations, we get:

$$
\tilde{\mathbf{h}}\left(\xi_{p}\right)=\operatorname{diag}\left\{\tilde{\mathbf{x}}_{d}^{p}\right\}^{-1} \tilde{\mathbf{r}}^{p}
$$

where $\boldsymbol{\xi}_{\boldsymbol{p}}=\left[\xi_{1}, \xi_{2}, \ldots, \xi_{P}\right]$ is a vector representing pilot frequencies and $P$ are total number of pilots. $\tilde{\mathbf{h}}\left(\xi_{p}\right)=\left[\tilde{h}\left(\xi_{1}\right), \tilde{h}\left(\xi_{2}\right), \ldots, \tilde{h}\left(\xi_{P}\right)\right]^{T}$, $\tilde{\mathbf{r}}^{p}=\left[\tilde{r}\left(\xi_{1}\right), \tilde{r}\left(\xi_{2}\right), \ldots, \tilde{r}\left(\xi_{P}\right)\right]^{T}$ and $\tilde{\mathbf{x}}_{d}^{p}=$ $\left[\tilde{x}\left(\xi_{1}, d\right), \tilde{x}\left(\xi_{2}, d\right), \ldots, \tilde{x}\left(\xi_{P}, d\right)\right]^{T}$ represent the channel transfer function, received and transmitted data corresponding to the desired user $d$ at pilot sub-carriers respectively. The notation $\operatorname{diag}\{\cdot\}$ represents a matrix formed by putting elements of the vector at the diagonal entries of the matrix with zeros at all off-diagonal entries. Estimates $\tilde{\mathbf{h}}\left(\xi_{p}\right)$ are then linearly interpolated to find the channel transfer function at data locations, and the interpolated vector can be expressed as $\tilde{\mathbf{h}}$. This is then used to cancel the channel effect on the receive signal, which can be presented as:

$$
\tilde{\mathbf{r}}_{e}=\operatorname{diag}\{\tilde{\mathbf{h}}\}^{-1} \tilde{\mathbf{r}}
$$

where $\tilde{\mathbf{r}}_{e}=\left[\tilde{r}_{e}(1), \tilde{r}_{e}(2), \ldots, \tilde{r}_{e}(N)\right]^{T}$ can be directly decoded to retrieve the transmitted data for each symbol. For the next symbol, beamformer computes the error vector between transmitted and received pilot sequence of the desired user at the beamformer's output. Based on this error vector, MSE based adaptive algorithm is used to update the beamformer weights for the next OFDM symbol. The error vector can be expressed as:

$$
\tilde{\mathbf{e}}^{p}=\tilde{\mathbf{r}}^{p}-\tilde{\mathbf{x}}_{d}^{p}
$$

The error vector obtained is in the frequency domain whereas beamforming is performed in the time domain. Hence this error vector needs to be transformed into the 

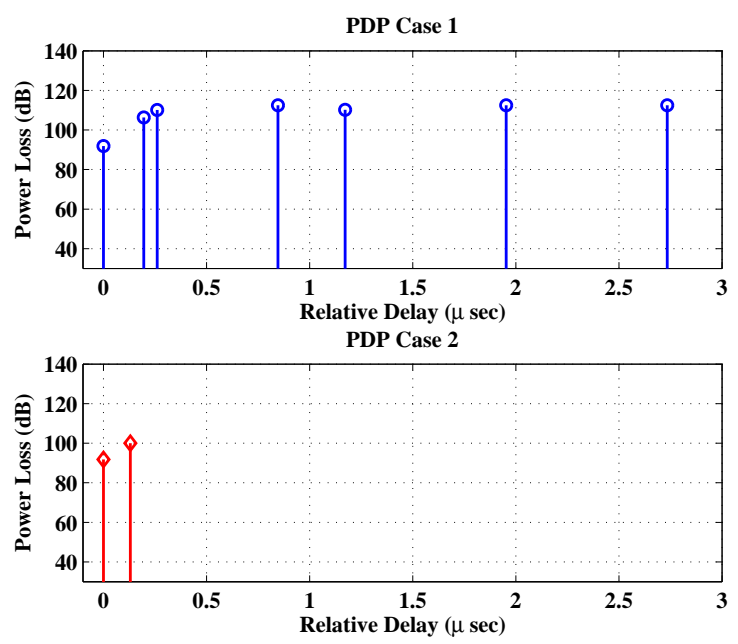

Figure 3: Power Delay Profile

time domain. This frequency-to-time error transform can be presented as:

$$
\mathbf{e}^{p}=\mathbf{F}_{p}^{H} \tilde{\mathbf{e}}^{p}
$$

Here $\mathbf{e}$ and $\tilde{\mathbf{e}}^{p}$ are the error vectors in time and frequency domain respectively. $\mathbf{F}_{p}$ can defined as the frequency-totime transform matrix.

$$
\mathbf{F}_{p}=\left[\begin{array}{ccc}
1 & \cdots & 1 \\
e^{-2 \pi\left(\xi_{1}\right)(1) / N} & \cdots & e^{-j 2 \pi\left(\xi_{P}\right)(1) / N} \\
\vdots & \ddots & \vdots \\
e^{-j 2 \pi\left(\xi_{1}\right)(N-1) / N} & \cdots & e^{-j 2 \pi\left(\xi_{P}\right)(N-1) / N}
\end{array}\right]
$$

After calculation of the error vector, we implement the widely used complex Least Mean Square (LMS) algorithm [3] to update the complex weights of the beamformer.

$$
\mathbf{w}(l+1)=\mathbf{w}(l)+2 \mu \mathbf{V}(l) \mathbf{e}^{p}(l)
$$

where $l=1,2, \ldots, L$ relates to the $l^{\text {th }}$ OFDM symbol with which weights will be computed for the next symbol and $\mu$ represents the LMS adaptive step size. This process is repeated until all desired user data has been extracted.

\section{Performance}

In this section we first outline transceiver parameters, discuss the mobile satellite channel model and then analyse the results. With regard to the transceiver parameters, a SIMO OFDM system with one transmit antenna and 5,9 receive antenna is used. Source data is modulated using QPSK with signal bandwidth and centre frequency being 5 $\mathrm{MHz}$ and $3 \mathrm{GHz}$ respectively. Total OFDM sub-carriers per symbol were 32 with sub-carrier 1,8,16,24,32 dedicated for pilot transmission. The desired user with respect to the satellite is at $40^{\circ}$ whereas two interference users are located at $-20^{\circ}$ and $70^{\circ}$. The power of interferers is set to $-10 \mathrm{dBW}$ and $\mu$ (LMS step size) is taken as 0.0007 . The multi-path

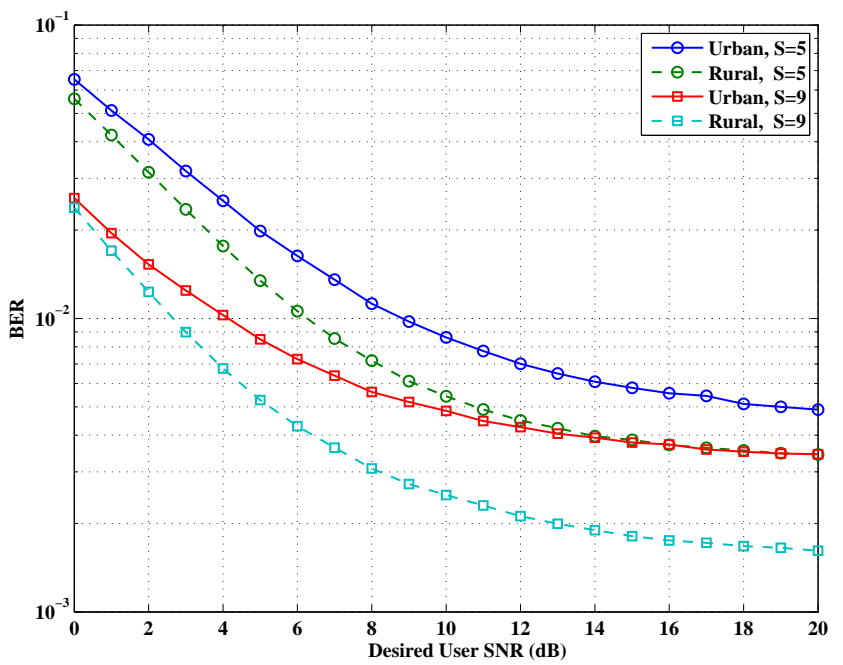

\begin{tabular}{|c|c|c|}
\hline Case 1 & \multicolumn{2}{|c|}{$\begin{array}{l}\text { Outdoor Rural } \\
K \text {-Factor }=10 \mathrm{~dB}\end{array}$} \\
\hline Tap Index & Relative Delay [ns] & Power Loss [dB] \\
\hline 1 & 0 & 91.9 \\
\hline 2 & 195.3 & 106.3 \\
\hline 3 & 260.4 & 110.1 \\
\hline 4 & 846.3 & 112.5 \\
\hline 5 & 1171.9 & 110.2 \\
\hline 6 & 1953.1 & 112.5 \\
\hline 7 & 2734.3 & 112.5 \\
\hline Case 2 & \multicolumn{2}{|c|}{$\begin{array}{c}\text { Outdoor Urban } \\
K \text {-Factor }=7 d B \\
\end{array}$} \\
\hline Tap Index & Relative Delay [ns] & Power Loss [dB] \\
\hline 1 & 0 & 91.8 \\
\hline 2 & 130.2 & 100 \\
\hline
\end{tabular}

Figure 4: BER vs desired user SNR for two environments with change in antenna elements, Mobile speed $=3 \mathrm{kmph}$.

Table 1: Channel Parameters

time selective channel model with parameters specific to mobile satellite scenario is modelled in order to analyse the system performance. The multi-path phenomenon is modelled as a linear Finite Impulse-Response filter with multiple taps and time selectivity of the channel is modelled using Jakes model [11]. Moreover we model the fading in case of satellite with Line-of-Sight (LOS) present as Rician. The channel parameters for two cases are presented in Table 1 and their respective Power Delay Profiles (PDPs) are plotted in Figure 3. These parameters were measured as part of the MAESTRO project [13].

In our first scenario, we chose pedestrian mobile speed with both cases to study the effect of the environment. Figure 4 presents the results and compares system performance in terms of Bit Error Rate (BER). We observe degradation in system performance as the user moves from rural to an urban area. This is attributed to a lower Rician K-factor and more frequency selective channel corresponding to the urban area. However, if the number of antenna elements are 


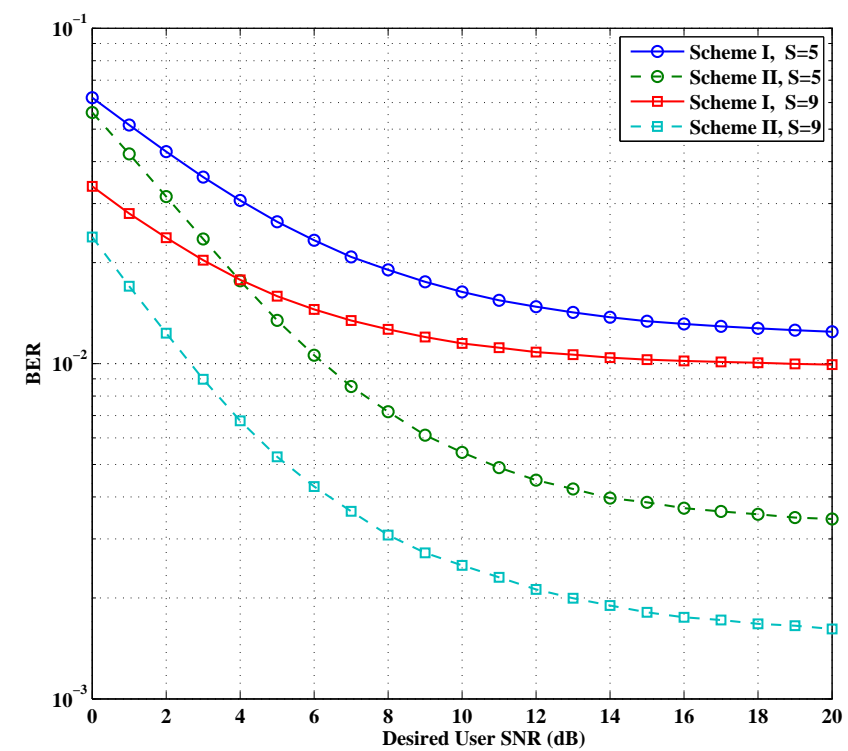

Figure 5: BER vs desired user SNR for two Schemes with change in antenna elements, Mobile speed $=3 \mathrm{kmph}$.

increased from 5 to 9 in the same scenario, an improvement in BER performance is observed. In practice, user speeds are higher and evaluation of performance in such context is needed. Moreover we will explore the interaction between beamforming and channel estimation. The beamformer task is to converge to the desired user whereas the channel estimation algorithm tries to alleviate the performance degradation due to the multi-path time selective channel. Hence we model two schemes; in scheme I channel estimation is not employed and all the processing is with the beamformer whereas in scheme II channel estimation aids the beamforming process before the desired user data is decoded. Figure 5 shows the results for both schemes when the user is moving with a speed of $3 \mathrm{kmph}$. It is observed that scheme II outperforms scheme I with 5 antenna elements. With 9 elements, the trend is the same however the performance difference is less between the two schemes. This highlights the role of channel estimation in terms of it's impact on system performance. Figure 6 presents results for a similar scenario but whilst keeping antenna elements constant and investigating the effect of schemes at different user speeds. We observe that at low speed, performance difference between the two schemes is large. However as the speed is increased to $60 \mathrm{kmph}$, the overall performance degrades but interestingly performance difference amongst the schemes drastically reduces. This indicates that with higher user speed, the Inter Carrier Interference becomes large and difficult for the beamformer and channel estimator to handle.

\section{Conclusion}

In this paper we propose an OFDM based hybrid architecture where satellite and existing mobile terrestrial networks amalgamate in a transparent fashion. Adaptive beamforming is then utilised to mitigate uplink CCI arising due to fre-

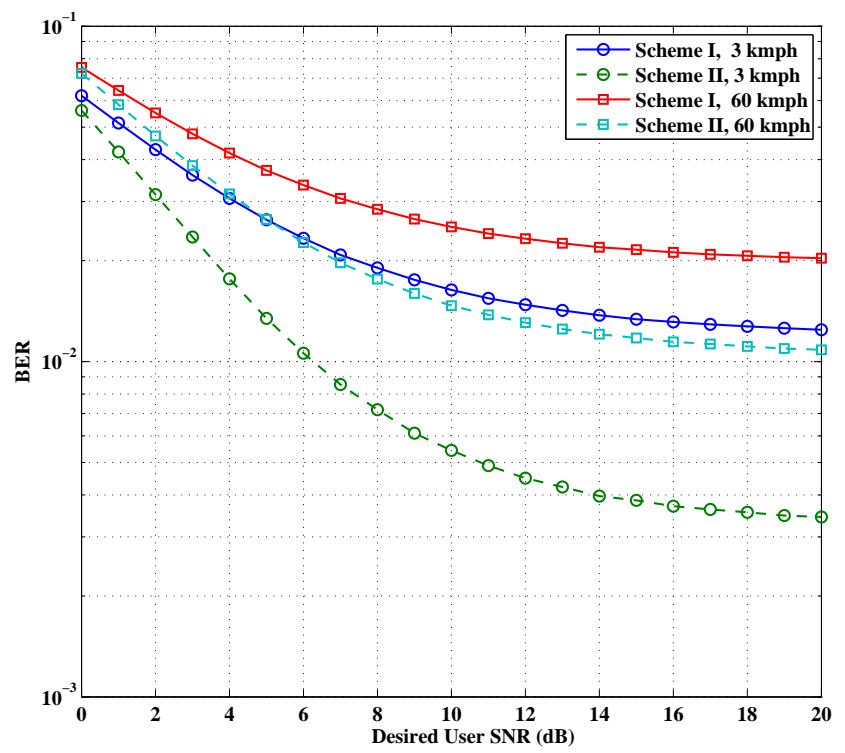

Figure 6: BER vs desired user SNR for two Schemes with change in mobile speed, Antenna elements $=5$.

quency reuse. We investigate this system in rural and urban environments with different schemes and mobile speeds to evaluate system performance. The simulation results show an improved system performance with increase in antenna elements. This was expected since more elements translate to better interference mitigation. In addition, as the user travels from rural to an urban area, degradation in performance is observed. We also explore the interplay between beamforming and channel estimation by applying two different schemes. We find that even though the beamformer tries to converge to the desired user, it is not able to cancel the channel effect. Thus channel estimator is essential and the beamformer alone cannot produce acceptable performance.

\section{Acknowledgements}

Ammar H. Khan would like to thank Centre for Communication Systems and Research (CCSR), United Kingdom and National University of Sciences and Technology (NUST), Pakistan for supporting him to undertake this work. Also, this work was partially conducted under the SATNEX II EU project to whom due acknowledgment is made.

\section{References}

[1] Y.-F. Chen and C.-S. Wang, "Adaptive antenna arrays for interference cancellation in OFDM communication systems with virtual carriers," vol. 56, no. 4, pp. 1837-1844, July 2007.

[2] S. Colieri, M. Ergen, A. Puri, and B. A, "A study of channel estimation in OFDM systems," in Proc. VTC 2002-Fall Vehicular Technology Conference 2002 IEEE 56th, vol. 2, 24-28 Sept. 2002, pp. 894-898.

[3] S. Haykin, Adaptive Filter Theory. $\quad$ Prentice, 1996. 
[4] P. D. Karabinis, "Systems and methods for terrestrial reuse of cellular satellite frequency spectrum," USA Patent 6684 057, January 27, 2004.

[5] P. D. Karabinis, S. Dutta, and W. W. Chapman, "Interference potential to MSS due to terrestrial reuse of satellite band frequencies." [Online]. Available: www.msvlp.com

[6] C. K. Kim, K. Lee, and Y. S. Cho, "Adaptive beamforming algorithm for OFDM systems with antenna arrays," vol. 46, no. 4, pp. 1052-1058, Nov. 2000.

[7] M. Lei and H. Harada, "Fast-convergence SMI adaptive beamforming based on frequency-to-time pilot transform for OFDM system," in Proc. WiCOM 2006.International Conference on Wireless Communications, Networking and Mobile Computing, 22-24 Sept. 2006, pp. 1-5.

[8] H. Liu and Q. Feng, "A blind adaptive beamforming for OFDM system," in Proc. Canadian Conference on Electrical and Computer Engineering, 1-4 May 2005, pp. 1208-1211.

[9] H. Matsuoka and H. Shoki, "Comparison of pre-FFT and post-FFT processing adaptive arrays for OFDM systems in the presence of co-channel interference," in Proc. 14th IEEE on Personal, Indoor and Mobile Radio Communications PIMRC 2003, vol. 2, 7-10 Sept. 2003, pp. 1603-1607.

[10] C.-H. Peng, K.-C. Huang, C.-Y. Chi, W.-K. Ma, and T.-H. Tsai, "A block-by-block blind post-FFT beamforming algorithm for multiuser OFDM systems based on subcarrier averaging," in Proc. IEEE 8th Workshop on Signal Processing Advances in Wireless Communications SPAWC 2007, 17-20 June 2007, pp. $1-5$.

[11] D. N. C. Tse and P. Viswanath, Fundamentals of Wireless Communication. Cambridge, U.K: Cambridge Univ. Press, 2005.

[12] D. Zheng and P. D. Karabinis, "Adaptive beamforming with interference supression in MSS with ATC." [Online]. Available: www.msvlp.com

[13] Dileverable D8-3 EC FP6/IST MAESTRO. [Online]. Available: http://maestro.gfi.fr/(accessed on: 26th March, 2009) 